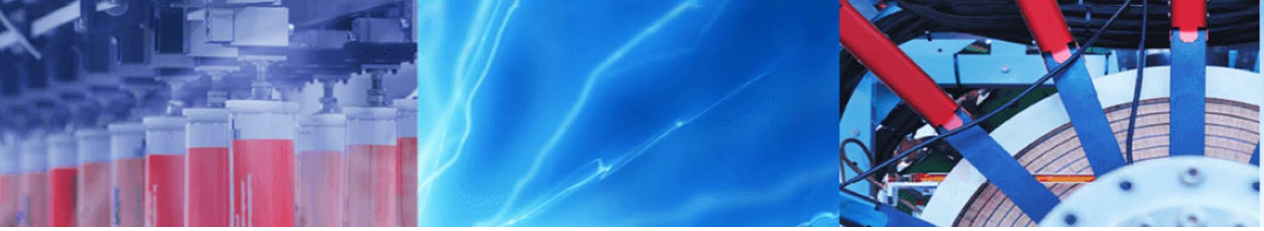

Research Article

\title{
A snail-shaped chaotic system with large bandwidth: dynamical analysis, synchronization and secure communication scheme
}

\author{
Khaled Benkouider ${ }^{1} \cdot$ Toufik Bouden $^{1} \cdot$ Mustak E. Yalcin ${ }^{2}$
}

Received: 15 October 2019 / Accepted: 29 April 2020 / Published online: 9 May 2020

(c) Springer Nature Switzerland AG 2020

\begin{abstract}
In this paper, a snail-shaped chaotic system with large bandwidth is first introduced, it contains two quadratic, one cubic and two quartic nonlinear terms. The new snail-shaped autonomous system can exhibit periodic, quasi-periodic and chaotic behaviors with the variations of its parameters. The major properties of the proposed model are discussed using equilibrium points, Kaplan-Yorke dimension, Lyapunov exponents spectrum and bifurcation diagrams. The feasibility of the snail-shaped system is verified by implementing an electronic circuit via Multisim software. The obtained results proving the complex chaotic behavior of the new system, which make it very desirable to use in many fields of engineering especially in secure communication. Also, synchronization between the snail-shaped chaotic system and the Rossler hyperchaotic system is achieved. Finally, a new simple secure communication scheme is developed based on the proposed system and using drive response synchronization in order to prove the success of the new system to complete the encryption/decryption process.
\end{abstract}

Keywords Chaotic system · Dynamical analysis · Lyapunov exponent · Bifurcation diagram · Synchronization · Circuit design

\section{Introduction}

In recent years, the study of chaotic systems become a very interest area of research because of its wide applications in many fields of science such as secure communication and cryptography [1-3].

Many chaotic systems have been proposed [4-6] since the first model introduced by the American meteorologist Edward Lorenz in 1963 [7]. The great technological development, especially in secure transmission making the designing of new chaotic systems a necessary need, but there is no uniform method to construct or to predict a chaotic behavior, For this reason all new systems are proposed as a mathematical models and verified using theoretical analysis and numerical simulations.
The main theoretical tool to identify a chaotic system is that of Lyapunov exponents, which are a quantity that characterizes the rate of divergence of close trajectories, a positive Lyapunov exponent indicate that the system generates a chaotic behavior. In addition, the real feasibility of the proposed systems is verified by designing its electronic circuits using simulation software or physical implementations.

In this work, a new snail-shaped chaotic system is first introduced which is very complex and has a large bandwidth; it contains eleven-terms with five nonlinearities making it able to generate rich dynamical behaviors. Dynamical properties are discussed theoretically and numerically. In addition, a comparative study between the proposed system and 30 recently proposed systems

Khaled Benkouider, benkouider.khaled@gmail.com; Toufik Bouden, bouden_toufik@yahoo.com; Mustak E. Yalcin, mustak.yalcin@itu.edu.tr | ${ }^{1}$ Non Destructive Testing Laboratory, Automatic Department, Jijel University, BP 98, 18000 Jijel, Algeria. ${ }^{2}$ Department of Electronics and Communication Engineering, Technical University, Istanbul, Turkey. 
is introduced. Then, an electronic circuit is implemented using Multisim software, which validate the theoretical analysis and proved the feasibility of the new system. Also, a synchronization between the new system and the classical Rossler hyperchaotic system is achieved using active control method. Finally, a secure communication scheme is developed using the drive response synchronization and the four signals generated by the new system. The obtained results confirm the effectiveness of the new snail-shaped chaotic system to complete the encryption/ decryption process in a secure communication scheme.

\section{The new chaotic attractor}

\subsection{Algebraic structure of the new system}

In this section, we announce a new 4D chaotic system with eleven terms, including five nonlinearities. The new system has four parameters and it is described by the following differential equations:

$\left\{\begin{array}{l}\dot{x}=a(y-x-z w) \\ \dot{y}=b x-x z^{3}-w^{3} \\ \dot{z}=x^{2}-z+w \\ \dot{w}=c x y z w-d w\end{array}\right.$

The new system generates a chaotic behavior for these values of parameters:

$a=4, b=4.8, c=0.5, d=0.5$

Some famous chaotic attractors have specifics names according to their shapes, among them: Butterfly-shaped attractor [8], torus-shaped attractor [9], funnel-shaped attractor [10] and so on. Our new system (1) exhibits a snail-shaped attractor as depicted in Fig. 1 for the parameters values as in (2). We can see that the new attractor in Fig. $1 \mathrm{~b}$ has a same shape as a snail that is shown in Fig. 2a.

\subsection{Sensitivity to initial conditions}

As we know, the main property of the chaotic systems is that of the sensitivity about the initial values. The high sensitivity to the initial conditions implies the existence of chaos. Figure 2 shows the high sensitivity of the proposed system (1) to a very little change in the initial values $\left( \pm 10^{-10}\right)$.

\subsection{Lyapunov exponents and Kaplan-Yorke dimension}

The two most important tools to characterize a chaotic behavior of a system are the Lyapunov exponents and the Kaplan-Yorke dimension.

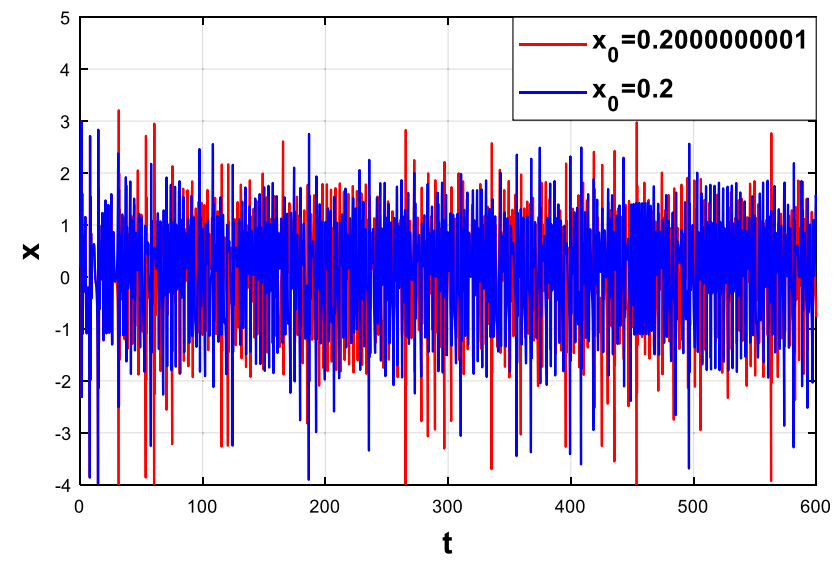

Fig. 2 Time series of the $x$ variable for $x_{0}=0.2$ and $x_{0}=0.2000000001$
Fig. 1 a A snail. b Chaotic snail-shaped attractor of the new system (1)

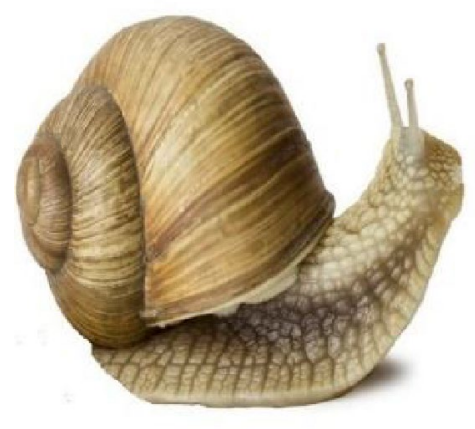

(a)

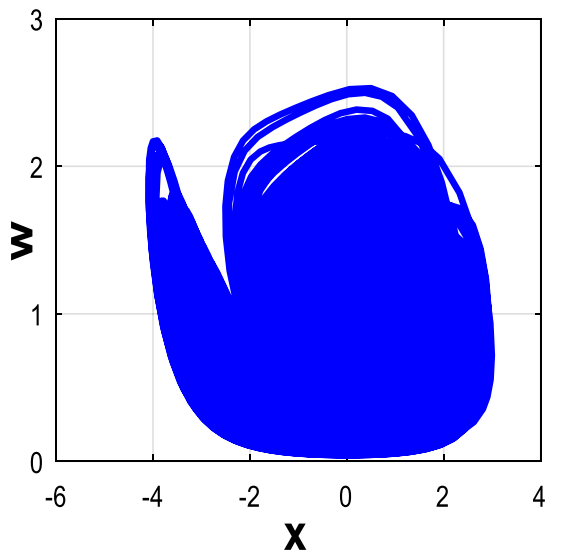

(b) 
As it is known, the Lyapunov exponent is a measure of exponential rates of convergence and divergence for an uncertainty on the trajectories initial points. When it is positive the uncertainty increases, which means divergence of trajectories (chaos). On the contrary, a negative exponent indicates convergence of the dynamical system trajectories [11].

Hence, a continuous dynamical system is chaotic, if it has at least one positive Lyapunov exponent.

The Lyapunov exponents of the proposed model (1) are shown in Fig. 3 as:

$L E_{1}=0.518, L E_{2}=0.000, L E_{3}=-0.795, L E_{4}=-4.722$

So, system (1) has one positive Lyapunov exponents. Hence, the new system is chaotic.

In addition, a chaotic attractor should have fractional dimension. For our proposed system (1), we used the Kaplan-Yorke dimension, which is a useful tool in order to determine the fractal dimension of the corresponding attractor [12], and it can be calculated by using the Lyapunov exponents as the following:

$K Y=2+\frac{L E_{1}+L E_{2}}{\left|L E_{3}\right|}$

$K Y=2+\frac{0.518}{|-0.795|}=2.652$

Since the Kaplan-Yorke dimension is fractal. So, the new model generates a complex chaotic behavior.

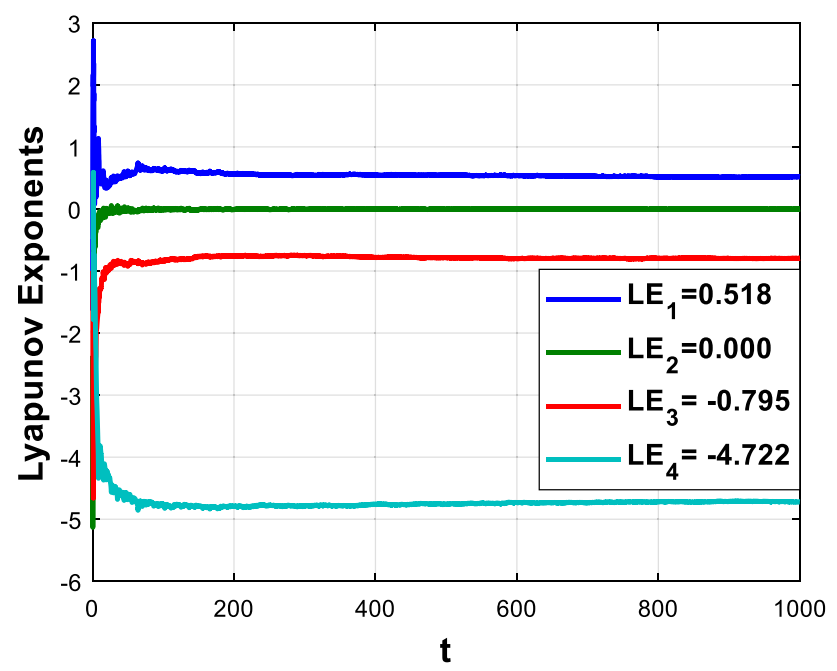

Fig. 3 Lyapunov exponents of the new system (1)

\subsection{Frequency spectrum and bandwidth}

Average normalised frequency spectrum of the new system (1) second state $y$ is depicted in Fig. 4. The bandwidth (BWT) of the new snail-shaped chaotic system is approximately equal to 15 , which is more than at least 30 chaotic systems reported in literature, as shown in Table 1.

\subsection{Comparison between the proposed chaotic system and 30 reported systems}

As we know:

1. Kaplan-Yorke dimension (KYD) is an index for characterizing the complexity and the unpredictability of a chaotic system, higher Kaplan-Yorke dimension implies higher complex chaotic behaviour [12].

2. The bandwidth (BWT) of a carrier signal generated by a chaotic system should be high in order to completely cover the masked information transmitted in a secure transmission scheme [13].

In this subsection, a comparison between the proposed snail-shaped chaotic system with 30 famous and recently reported chaotic systems is introduced using Kaplan-Yorke dimension and bandwidth. This comparison showed that the Kaplan-Yorke dimension of our system is higher than the most famous and novel chaotic systems. In addition, we can see from Table 1 that the bandwidth of system (1) is much higher than the other proposed systems. Therefore, the new snail-shaped chaotic system is more complex and more useful for secure communication than at least 30 reported systems as explained in Table 1.

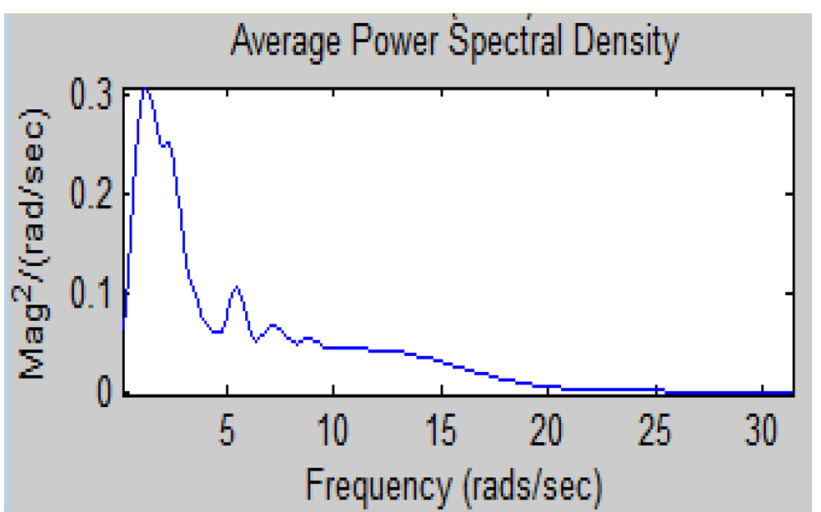

Fig. 4 Average power spectrum of the second state signal $y$ 
Table 1 Kaplan-Yorke dimension and bandwidth of 30 reported systems

\begin{tabular}{|c|c|c|c|}
\hline No. & System & KYD & BWT \\
\hline 1 & Lorenz [7] & 2.062 & 4 \\
\hline 2 & Chen [14] & 2.175 & 6 \\
\hline 3 & Su [15] & 2.044 & 5 \\
\hline 4 & Akgul [16] & 2.024 & 5 \\
\hline 5 & Zhang [17] & 2.025 & 5 \\
\hline 6 & Gholamin et al. [18] & 2.055 & 6 \\
\hline 7 & Lai et al. [19] & 2.196 & 6 \\
\hline 8 & Tuna et al. [20] & 2.191 & 4 \\
\hline 9 & Wu et al. [21] & 2.015 & 7 \\
\hline 10 & Volos et al. [22] & 2.175 & 2.5 \\
\hline 11 & Jay et al. [23] & 2.160 & 13 \\
\hline 12 & Sambas et al. [24] & 2.072 & 1.5 \\
\hline 13 & Yang et al. [25] & 2.112 & 4 \\
\hline 14 & Lassoued et al. [26] & 2.124 & 2 \\
\hline 15 & Vaidyanathan et al. [27] & 2.162 & 9 \\
\hline 16 & Lien et al. [28] & 2.163 & 3 \\
\hline 17 & Kapitaniak et al. [29] & 2.107 & 3 \\
\hline 18 & Xu et al. [30] & 2.074 & 2 \\
\hline 19 & Idowu et al. [31] & 2.236 & 2 \\
\hline 20 & Jinjie et al. [32] & 2.185 & 9 \\
\hline 21 & Lai et al. [33] & 2.091 & 8 \\
\hline 22 & Sahin et al. [34] & 2.579 & 2 \\
\hline 23 & Zhou et al. [35] & 2.062 & 4 \\
\hline 24 & Vaidyanathan et al. [36] & 2.063 & 5 \\
\hline 25 & Tasikmalaya et al. [37] & 2.066 & 7 \\
\hline 26 & Wang et al. [38] & 2.032 & 4 \\
\hline 27 & Vaidyanathan et al. [39] & 2.213 & 2 \\
\hline 28 & Yanmin et al. [40] & 2.276 & 10 \\
\hline 29 & Zheng [41] & 2.594 & 2 \\
\hline 30 & Benkouider et al. [42] & 2.280 & 13 \\
\hline 31 & System (1) & 2.652 & 15 \\
\hline
\end{tabular}

\section{Dynamical analysis of the new system}

\subsection{Dissipativity}

The divergence of the system (1) is calculated by the following formula:

$\nabla V=\frac{\partial \dot{x}}{\partial x}+\frac{\partial \dot{y}}{\partial y}+\frac{\partial \dot{z}}{\partial z}+\frac{\partial \dot{w}}{\partial w}=-1-1-d+c x y z$

Hence,

$\nabla V=-2-d+c x y z$

Since, $c x y z<d+2$ so, $\nabla V<0$. Hence, the new $4 D$ system is dissipative and all system (1) orbits are ultimately confined to a specific subset of zero volume, and the asymptotic motion settles onto an attractor.

\subsection{Equilibrium points and stability}

The equilibrium points of the new system (1) are founded by solving the following set of equations:

$$
\left\{\begin{aligned}
a(y-x-z w) & =0 \\
b x-x z^{3}-w^{3} & =0 \\
x^{2}-z+w & =0 \\
c x y z w-d w & =0
\end{aligned}\right.
$$

System (1) has a unique equilibrium point $\mathrm{S}$ :

$S=[0,0,0,0]$

The stability of system (1) around this equilibrium point is analysed by using the below Jacobian matrix:

$$
J_{x, y, z, w}=\left[\begin{array}{cccr}
-a & a & -a w & -a z \\
b-z^{3} & 0 & -3 x z^{2} & -3 w^{2} \\
2 x & 0 & -1 & 1 \\
c y z w & c x z w & c x y w & -d+c x y z
\end{array}\right]
$$

For the equilibrium point $\mathrm{S}$, the Jacobian matrix become as follows:

$$
J_{S}=\left[\begin{array}{cccr}
-a & a & 0 & 0 \\
b & 0 & 0 & 0 \\
0 & 0 & -1 & 1 \\
0 & 0 & 0 & -d
\end{array}\right]
$$

In order to find the eigenvalues of the Jacobian matrix (10), and for the parameters values as in (2), we are solving the corresponding characteristic equation:

$\lambda^{4}+5.5 \lambda^{3}-12.7 \lambda^{2}-26.8 \lambda-9.6=0$

The eigenvalues are found as:

$\lambda_{1}=-6.82, \lambda_{2}=-1, \lambda_{3}=-0.5, \lambda_{4}=2.82$

Since $\lambda_{1,2,3}<0$ and $\lambda_{4}>0$ so, the equilibrium point $S$ is unstable.

\subsection{Bifurcation analysis}

\subsubsection{Fix $b=4.8, c=0.5, d=0.5$, and vary $a$}

The Bifurcation diagram of the new system (1) with respect to parameter $a$ is depicted in Fig. 5. Also, the Lyapunov exponents spectrum is shown in Fig. 6 . We can see that 


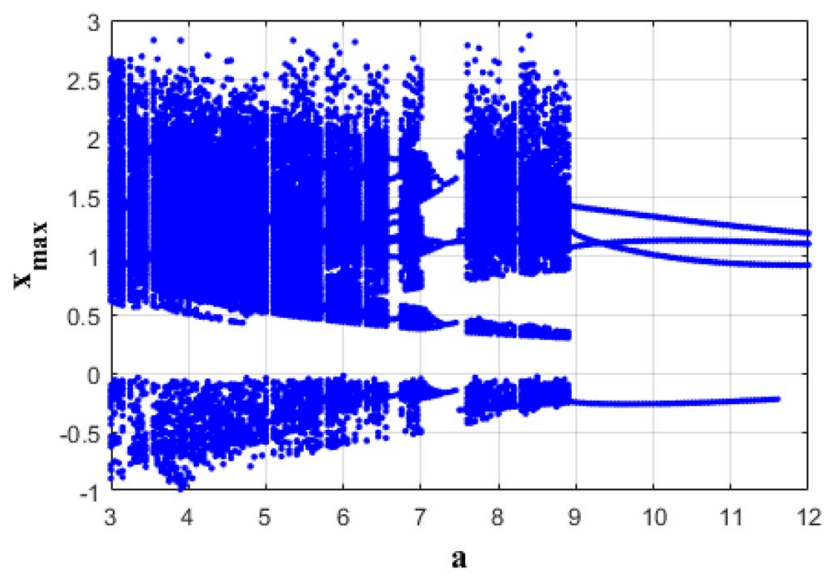

Fig. 5 Bifurcation diagram of the system (1) first state versus parameter a
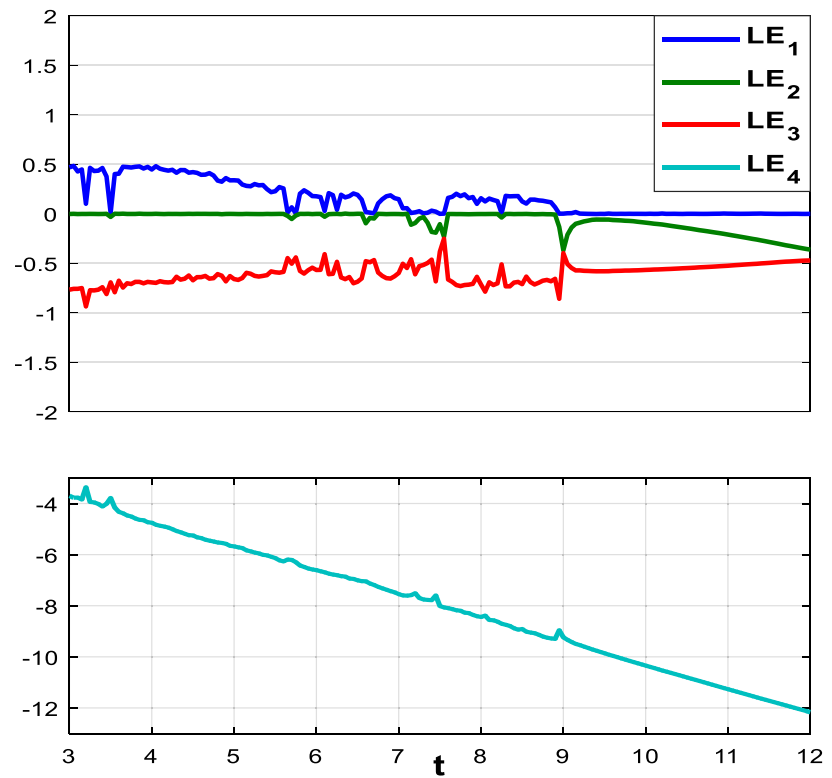

Fig. 6 Lyapunov exponents of the system (1) versus parameter a the Bifurcation diagram and the spectrum of Lyapunov exponents are completely compatible.

From Figs. 5 and 6, we note that the new system can exhibit different dynamical behaviors with the variation of parameter $a$, as it is summarized below:

$$
\begin{aligned}
& \text { - If } a=11 \in[3.15,3.25[\cup] 3.40,3.55[\cup] 5.60,5.80[\cup \\
& \text { - } \quad] 6.20,6.30[\cup] 6.55,6.80[\cup] 7.00,7.60[\cup \\
& \\
&
\end{aligned}
$$

The corresponding Lyapunov exponents are: $L E_{1}=0.00, L E_{2}=-0.16, L E_{3}=-0.75, L E_{4}=-11.0950$, system (1) generates periodic orbits such as depicted in Fig. 7.

- If $a=9.5 \in[9.2,10.5]$, the corresponding Lyapunov exponents are:

$$
L E_{1}=0.00, L E_{2}=0.00, L E_{3}=-0.75, L E_{4}=-9.67
$$

So, system (1) generates quasi-periodic orbits such as depicted in Fig. 8.

$a=4 \in[3.00,3.15[\cup] 3.25,3.40[\cup] 3.55,5.6[\cup$

- If $\quad] 5.8,6.2[\cup] 6.3,6.55[\cup] 6.8,7[\cup$

] $7.6,8.2[\cup] 8.4,8.8]$

The corresponding Lyapunov exponents are:

$$
L E_{1}=0.52, L E_{2}=0.00, L E_{3}=-0.79, L E_{4}=-4.72
$$

So, system (1) generates chaotic attractor such as depicted in Fig. 9.

\subsubsection{Fix $a=4, c=0.5, d=0.5$, and vary $b$}

The Bifurcation diagram of the new system (1) with respect to parameter $b$ is depicted in Fig. 10. Also, the Lyapunov exponents spectrum is shown in Fig. 11. We can see that the Bifurcation diagram and the spectrum of Lyapunov exponents are completely compatible.

From Figs. 10 and 11, we note that the new system can exhibit periodic and chaotic behaviors with the variation of parameter $b$, as it is summarized below:
Fig. 7 Phase portraits of the periodic orbits
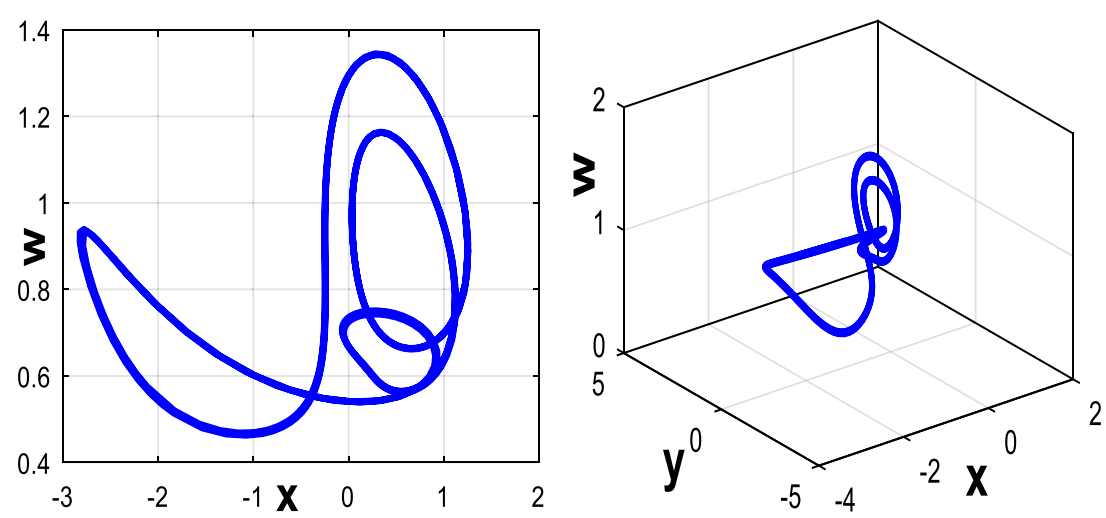

SN Applied Sciences A SPRINGER NATURE journa 
Fig. 8 Phase portraits of the quasi-periodic orbits

Fig. 9 Phase portraits of the snail-shaped chaotic attractor
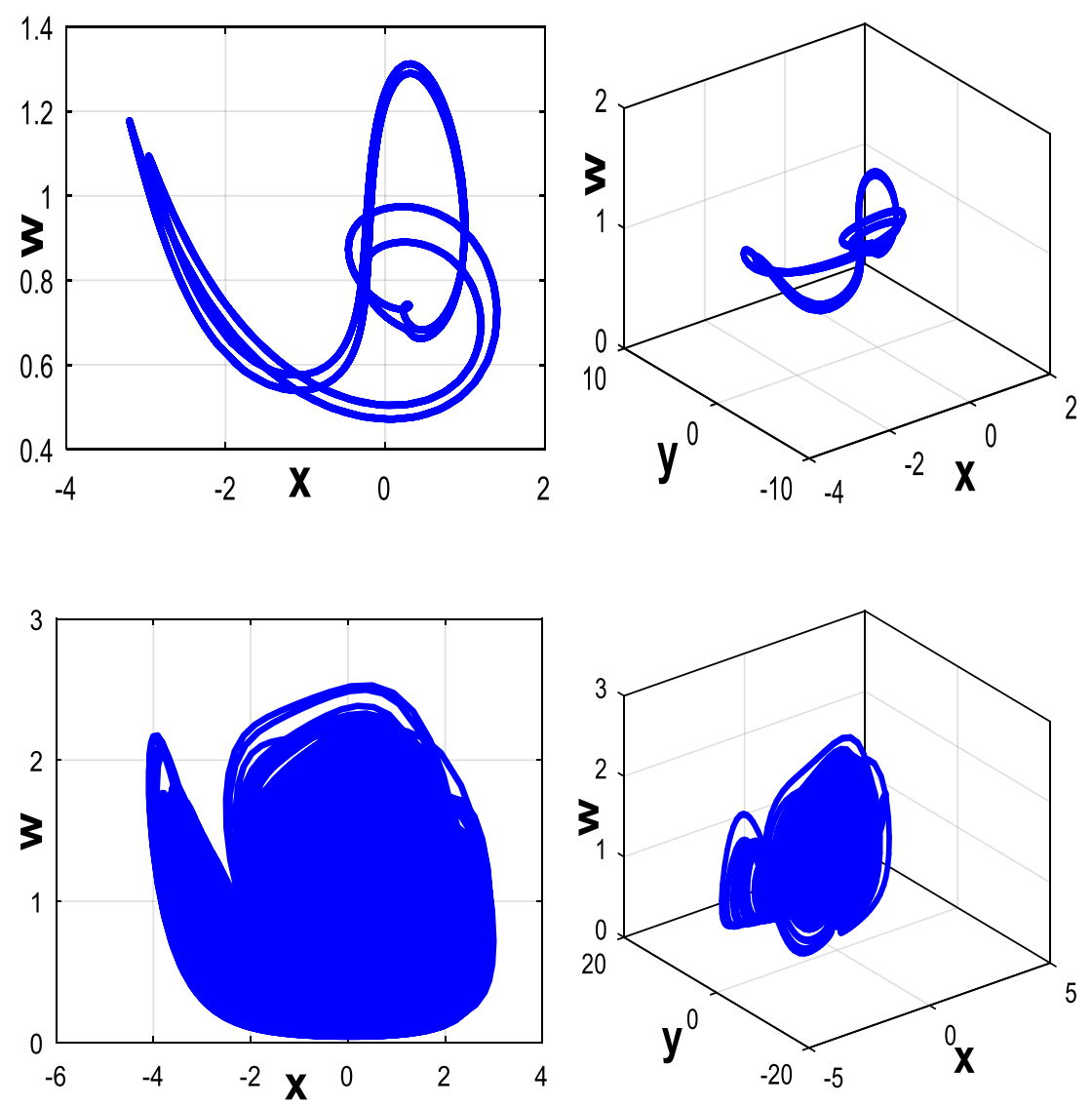

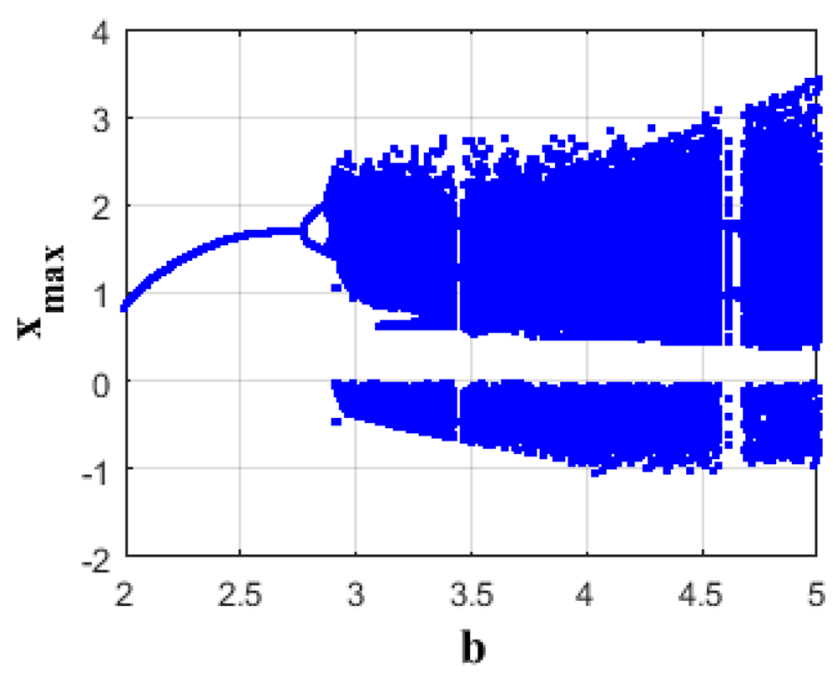

Fig. 10 Bifurcation diagram of the system (1) first state versus parameter b

- If $b=2.5 \in[2.00,2.90[\cup] 3.43,3.46[\cup] 4.60,4.66]$, the corresponding Lyapunov exponents are:

$L E_{1}=0.00, L E_{2}=-0.48, L E_{3}=-0.50, L E_{4}=-4.02$

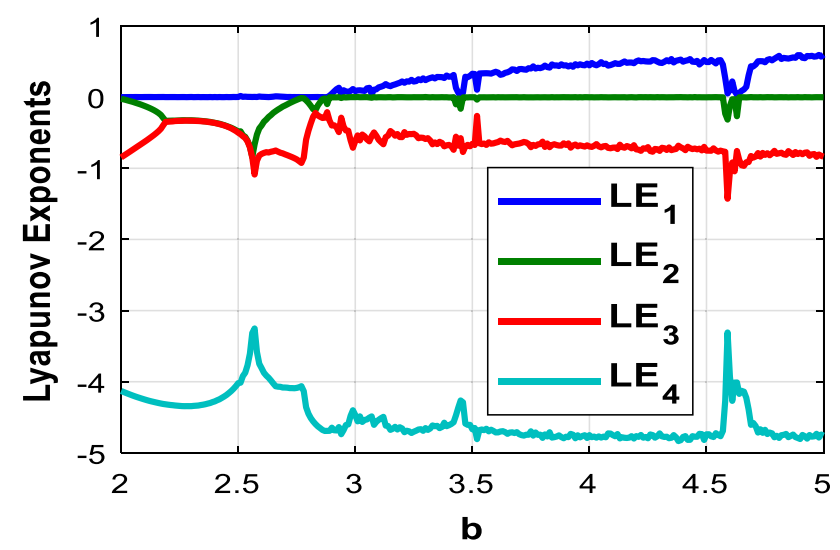

Fig. 11 Lyapunov exponents of the system (1) versus parameter b

So, system (1) generates periodic orbits such as depicted in Fig. 12.

- If $b=4 \in[2.90,3.43[\cup] 3.46,4.60[\cup] 4.66,5.00]$, the corresponding Lyapunov exponents are:

$L E_{1}=0.45, L E_{2}=0.00, L E_{3}=-0.69, L E_{4}=-4.75$

So, system (1) generates chaotic attractor such as depicted in Fig. 13 
Fig. 12 Phase portraits of the periodic orbits
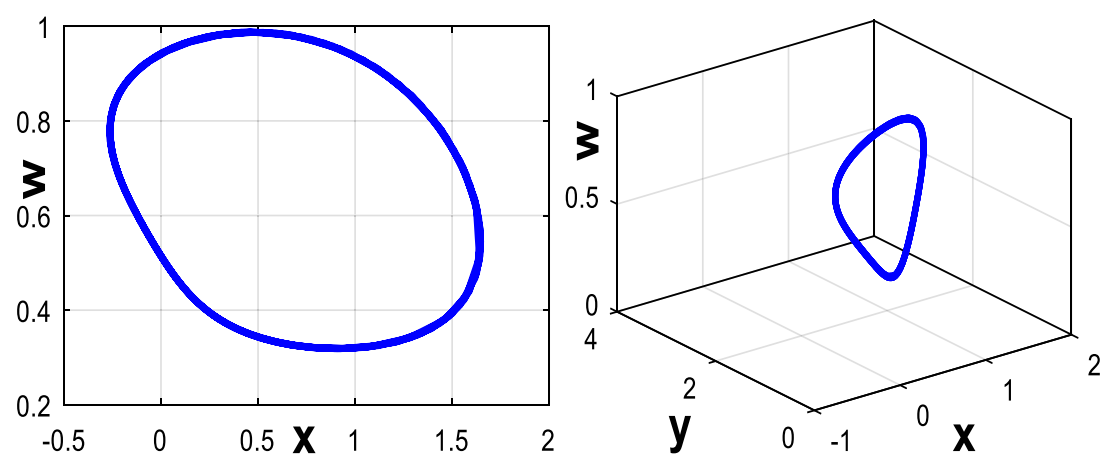

Fig. 13 Phase portraits of the chaotic attractor
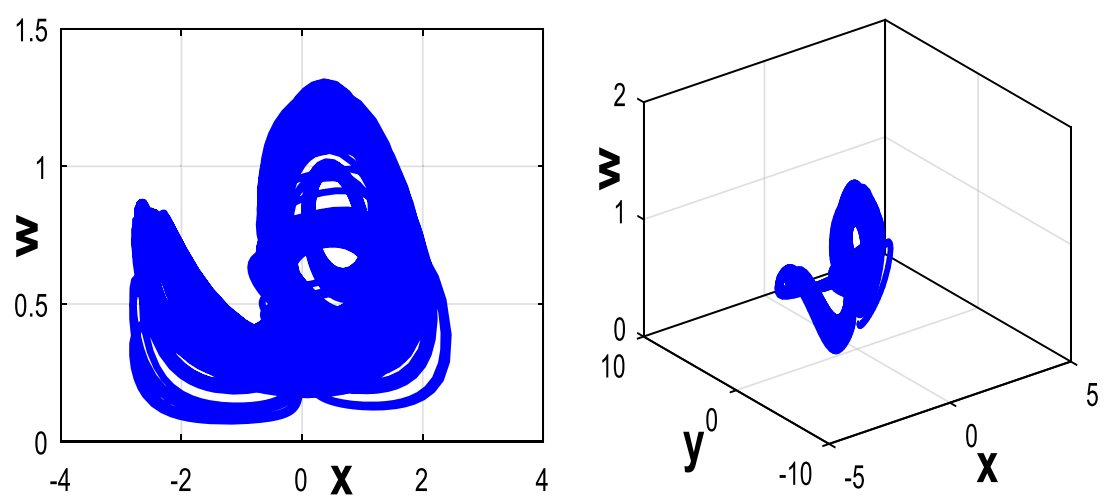

\section{Electronic circuit implementation}

In order to verify the feasibility of the proposed chaotic attractor, an electronic circuit is designed using Multisim software.

According to the dynamical equations of the new 4D system (1), the corresponding equations of the electronic circuit are given by:

$\left\{\begin{array}{l}\dot{x}=\frac{1}{R_{1} C_{1}} y-\frac{1}{R_{2} C_{1}} x-\frac{1}{R_{3} C_{1}} z w \\ \dot{y}=\frac{1}{R_{4} C_{2}} x-\frac{1}{R_{5} C_{2}} x z^{3}-\frac{1}{R_{6} C_{2}} w^{3} \\ \dot{z}=\frac{1}{R_{7} C_{3}} x^{2}-\frac{1}{R_{8} C_{3}} z+\frac{1}{R_{9} C_{3}} w \\ \dot{w}=\frac{1}{R_{10} C_{4}} x y z w-\frac{1}{R_{11} C_{4}} w\end{array}\right.$

The schematic of the circuit is shown in Fig. 14.

The values of the electronic circuit elements are chosen as follows:

$$
\left\{\begin{aligned}
R_{1} & =R_{2}=R_{3}=42.105 \mathrm{k} \Omega \text { (Quasi - periodic) } \\
R_{1} & =R_{2}=R_{3}=36.363 \mathrm{k} \Omega \text { (Periodic) } \\
R_{1} & =R_{2}=R_{3}=100 \mathrm{k} \Omega \text { (Chaotic) } \\
R_{4} & =83.334 \mathrm{k} \Omega \\
R_{5} & =R_{6}=R_{7}=R_{8}=R_{9}=400 \mathrm{k} \Omega \\
R_{10} & =R_{11}=800 \mathrm{k} \Omega \\
R_{12} & =R_{13}=R_{14}=R_{15}=R_{16}=R_{17}=100 \mathrm{k} \Omega \\
C_{1} & =C_{2}=C_{3}=C_{4}=1 \mathrm{nF}
\end{aligned}\right.
$$

Figures 15,16 and 17 show respectively the snailshaped attractor, the periodic orbit and the quasi-periodic orbit of the new system (1) via Multisim software which is consistent with the Matlab simulations.

All this results proved the real feasibility of the proposed snail-shaped chaotic system (1).

\section{Synchronization of the new chaotic system with Rossler hyperchaotic system}

In this section, synchronization between system (1) and the famous Rossler hyperchaotic system (15) is studied and achieved via active control. We are proved that the proposed chaotic system can be synchronized not only with chaotic systems but also with hyperchaotic systems, which make it 
Fig. 14 The circuit schematic

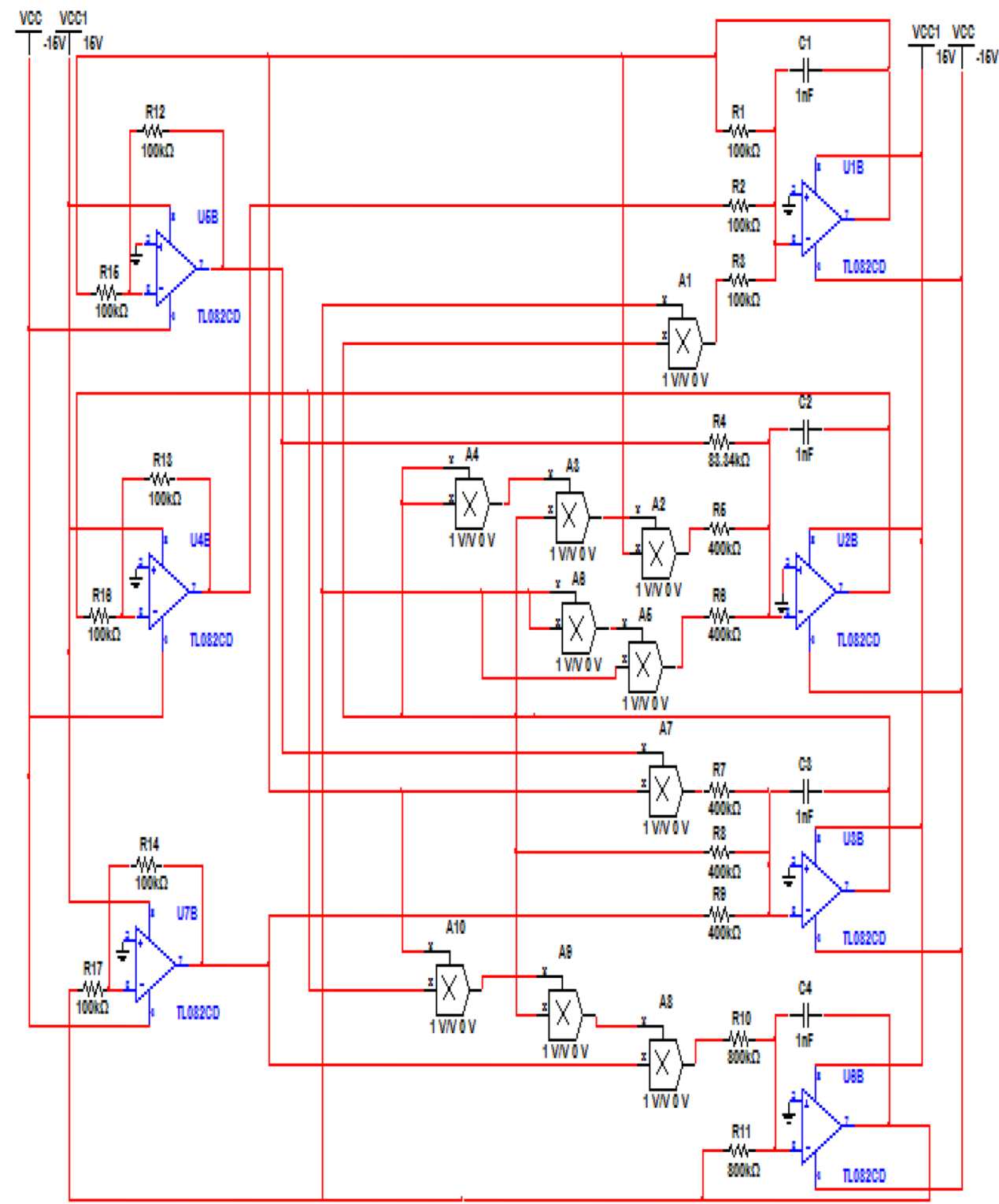

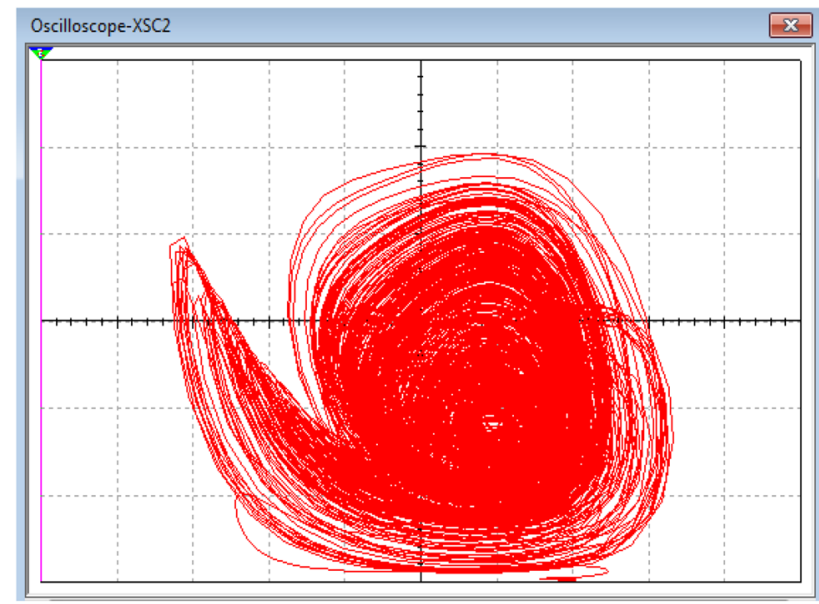

Fig. 15 Multisim result of the snail-shaped chaotic attractor

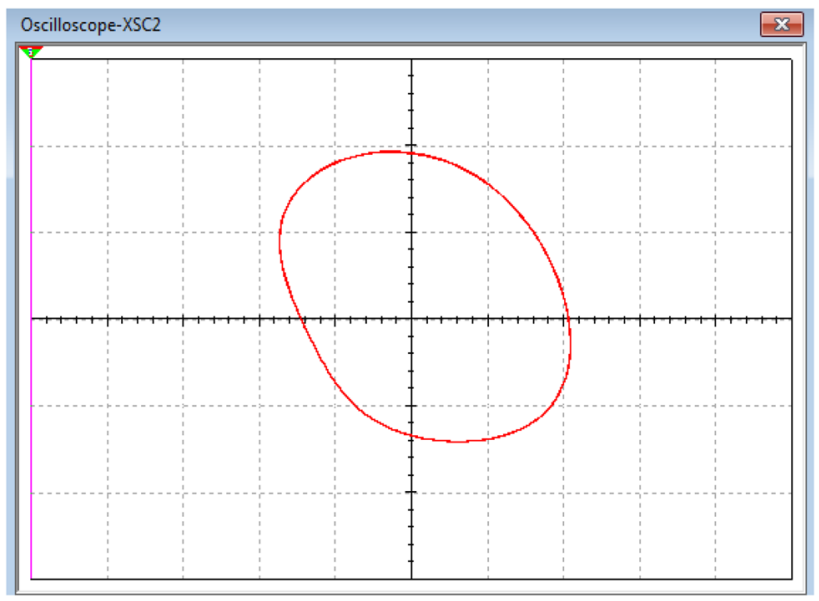

Fig. 16 Multisim result of the periodic orbit

\section{SN Applied Sciences}




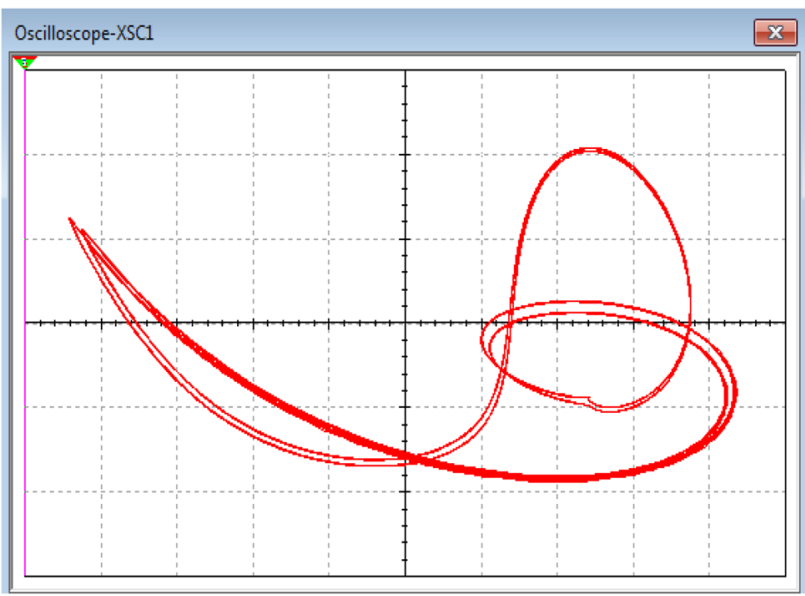

Fig. 17 Multisim result of the quasi-periodic orbit

very useful in different fields of science especially in secure transmission.

The classical Rossler hyperchaotic system is described as follows:

$\left\{\begin{aligned} \dot{x} & =f y+g x \\ \dot{y} & =3+y z \\ \dot{z} & =-y-w \\ \dot{w} & =x+z+h w\end{aligned}\right.$

Where,

$f=-0.5, g=0.05, h=0.25$

With these values of parameters, Rossler system has two positive Lyapunov exponents:

$L E_{1}=0.109$ and $L E_{2}=0.024$
So, system (15) exhibits a hyperchaotic behavior as depicted in Fig. 18.

\subsection{Control functions design}

The Rossler system (15) is considered as a master system and it is described as follows:

$\left\{\begin{array}{l}\dot{x}_{m}=f y_{m}+g x_{m} \\ \dot{y}_{m}=3+y_{m} z_{m} \\ \dot{z}_{m}=-y_{m}-w_{m} \\ \dot{w}_{m}=x_{m}+z_{m}+h w_{m}\end{array}\right.$

The new system (1) is considered as a slave system and it is described as follows:

$\left\{\begin{array}{l}\dot{x}_{s}=a\left(y_{s}-x_{s}-z_{s} w_{s}\right)+u_{x} \\ \dot{y}_{s}=b x_{s}-x_{s} z_{s}^{3}-w_{s}^{3}+u_{y} \\ \dot{z}_{s}=x_{s}^{2}-z_{s}+w_{s}+u_{z} \\ \dot{w}_{s}=c x_{s} y_{s} z_{s} w_{s}-d w_{s}+u_{w}\end{array}\right.$

Where $u_{x}, u_{y}, u_{z}$ and $u_{w}$ are the active control functions to be found. The state errors between the master and the slave systems are defined as:

$e_{x}=x_{s}-x_{m}, e_{y}=y_{s}-y_{m}, e_{z}=z_{s}-z_{m}, e_{w}=w_{s}-w_{m}$

Hence, the dynamical of state errors is described by the following equations:

$\left\{\begin{array}{l}\dot{e}_{x}=a y_{s}-a x_{s}-a z_{s} w_{s}-f y_{m}-g x_{m}+u_{x} \\ \dot{e}_{y}=b x_{s}-x_{s} z_{s}-w_{s}-3-y_{m} z_{m}+u_{y} \\ \dot{e}_{z}=x_{s}^{2}-z_{s}+w_{s}+y_{m}+w_{m}+u_{z} \\ \dot{e}_{w}=c x_{s} y_{s} z_{s} w_{s}-d w_{s}-x_{m}-z_{m}-h w_{m}+u_{w}\end{array}\right.$
Fig. 18 Rossler hyperchaotic attractor
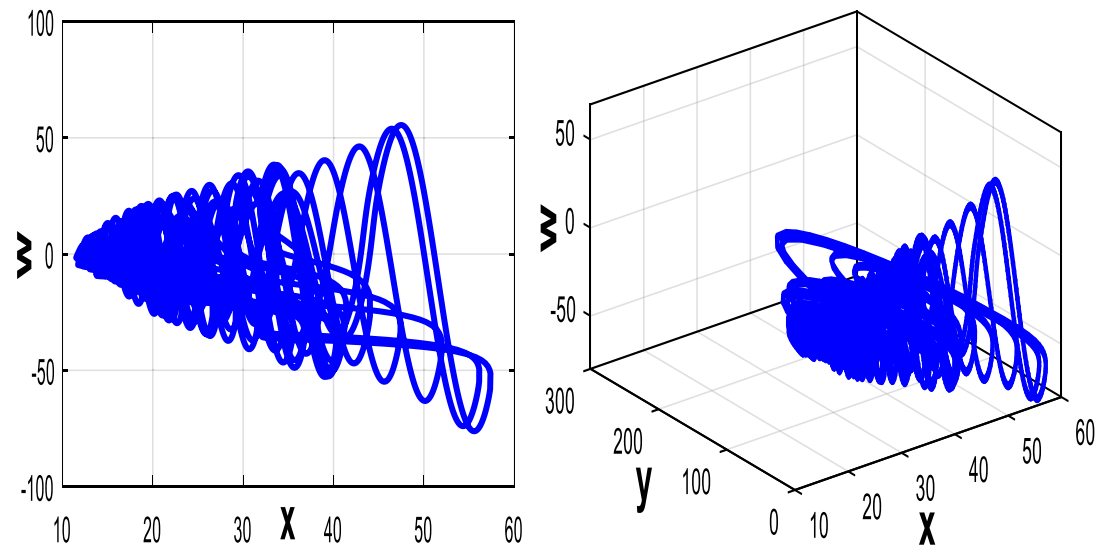

SN Applied Sciences 
Theorem If we choose the active control functions as the following:

$\left\{\begin{array}{l}u_{x}=(a+g) x_{m}-(a-f) y_{m}+a z_{s} w_{s}-a e_{y}+(a-1) e_{x} \\ u_{y}=x_{s} z_{s}^{3}+w_{s}^{3}+3+y_{m} z_{m}-b x_{m}-b e_{x}-e_{y} \\ u_{z}=-x_{s}^{2}+z_{m}-y_{m}-2 w_{m}-e_{w} \\ u_{w}=-c x_{s} y_{s} z_{s} w_{s}+(d+h) w_{m}+x_{m}+z_{m}+(d-1) e_{w}\end{array}\right.$

The dynamical of state errors will be converge asymptotically to zero. Hence, the new chaotic system (1) and the Rossler hyperchaotic system (15) will be synchronized.

Proof Considering the active control functions defined in (21), the dynamical of state errors (20) can be rewritten as:

$\left\{\begin{array}{c}\dot{e}_{x}=-e_{x} \\ \dot{e}_{y}=-e_{y} \\ \dot{e}_{z}=-e_{z} \\ \dot{e}_{w}=-e_{w}\end{array}\right.$

Therefore, $\left[\begin{array}{c}\dot{e}_{x} \\ \dot{e}_{y} \\ \dot{e}_{z} \\ \dot{e}_{w}\end{array}\right]=\left[\begin{array}{cccc}-1 & 0 & 0 & 0 \\ 0 & -1 & 0 & 0 \\ 0 & 0 & -1 & 0 \\ 0 & 0 & 0 & -1\end{array}\right]\left[\begin{array}{c}e_{x} \\ e_{y} \\ e_{z} \\ e_{w}\end{array}\right]$

All eigenvalues of the state matrix are negative, so, according to Routh-Hurwitz criterion; the errors dynamics are stable which ensure synchronization between the master system (15) and the slave system (1).

\subsection{Numerical simulation results}

Matlab/Simulink software is used to implement the control laws in order to synchronize the two systems.

The parameters values as in (2) and the initial conditions $[20,-7,0.2,0.2]$ are chosen for the proposed system (1) and the parameters values as in (16) and the initial conditions [14, 2, -17, 7] are chosen for the Rossler system (15).

Figures 19 and 20 show that the two systems are synchronized and the state errors converge to zero exponentially, which proved the effectiveness of the designed control functions to synchronize the proposed snail-shaped chaotic system (1) and the hyperchaotic system of Rossler (15).
Fig. 19 Coordinates times series of the master and the slave systems
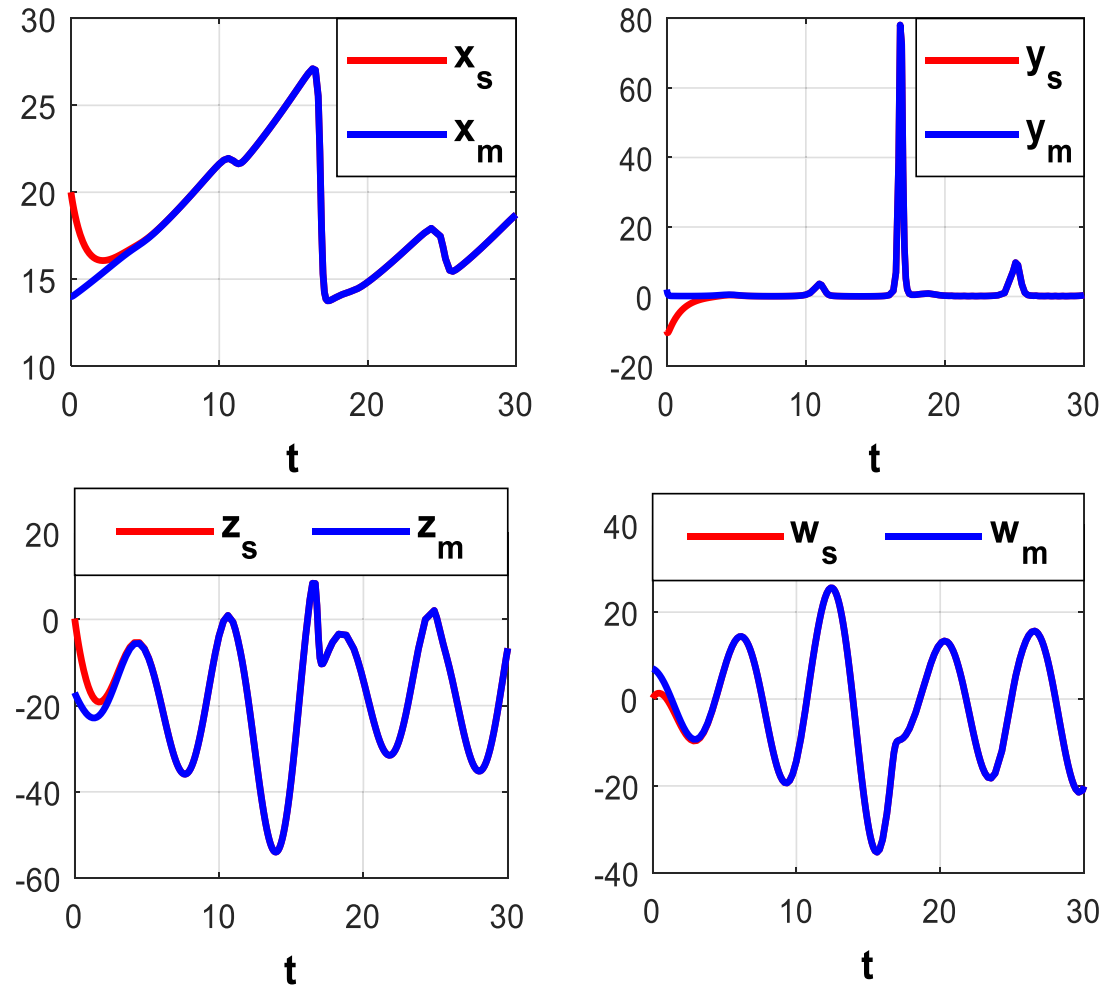


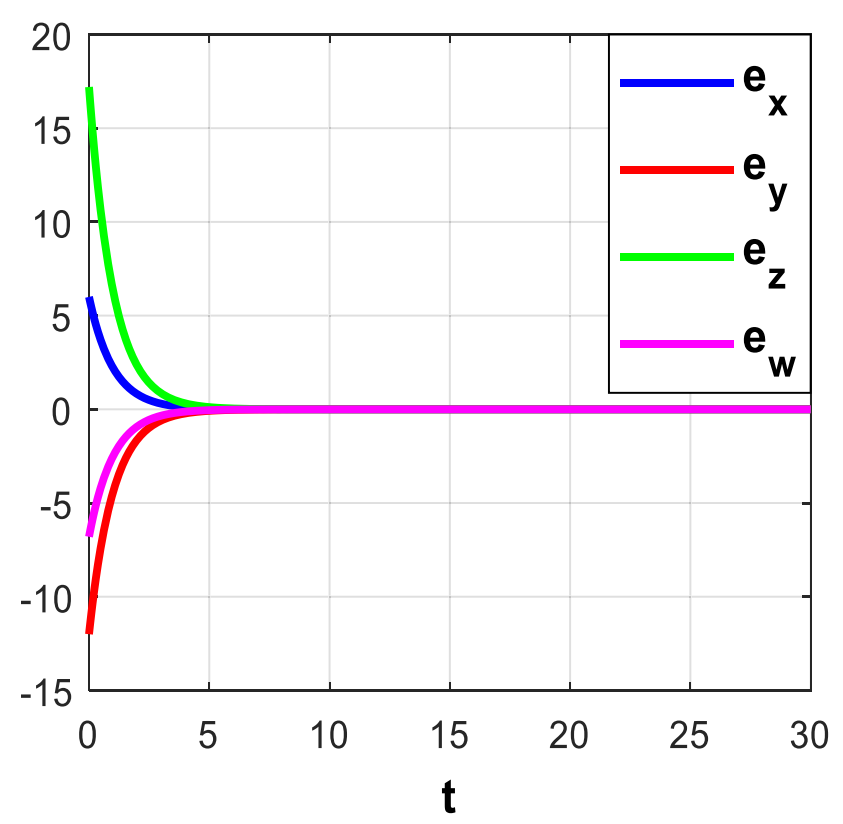

Fig. 20 Errors time series between the master and the slave systems

\section{Secure transmission scheme based on the proposed chaotic system}

In this section, a new secure communication scheme is developed for the new snail-shaped chaotic system (1) using the drive response synchronization which has the advantage of strong self-synchronization and the use of only one coordinate to achieve synchronization.

The new scheme is tested using Matlab/Simulink software, simulation results showing the success of the proposed system to complete the encryption/decryption process.

Matlab/Simulink block diagram of the new secure communication scheme is depicted in Fig. 21.

\subsection{Drive response synchronization}

Consider the new snail-shaped chaotic system (1) as master system; the slave system is described by the following equations:

$\left\{\begin{array}{l}\dot{x}_{s}=a\left(y_{s}-x_{s}-z_{s} w_{s}\right) \\ \dot{y}_{s}=b x_{s}-x_{s} z_{s}^{3}-w_{s}^{3} \\ \dot{z}_{s}=x_{s}^{2}-z_{s}+w_{s} \\ \dot{w}_{s}=c x_{s} y_{s} z_{s} w_{s}-d w_{s}\end{array}\right.$

By choosing the second state variable of the master system $y_{m}$ as driving variable in order to lead the slave system (24) to achieve synchronization with master system (1), the slave system can be rewritten as follows:

$\left\{\begin{array}{l}\dot{x}_{s}=a\left(y_{m}-x_{s}-z_{s} w_{s}\right) \\ \dot{z}_{s}=x_{s}^{2}-z_{s}+w_{s} \\ \dot{w}_{s}=c x_{s} y_{m} z_{s} w_{s}-d w_{s}\end{array}\right.$

Simulation results depicted in Figs. 22, 23 and 24 showing the synchronization errors between the master coordinates and the slave coordinates, it can be seen that all synchronization errors converge to zero after few second ( $5 \mathrm{~s}$ ), which confirm the success of the drive response method to synchronize the two identical master and slave snail-shaped chaotic systems starting from different initial values.

\subsection{Encryption/decryption process}

Using the drive response synchronization, a new encryption/decryption process is developed for the new snailshaped system as depicted in Fig. 21b, c.

The encrypted message $E M$ that will be transmitted is obtained by switching between two different encryption processes according to the value of the master system first state $x_{m}$ as the following:

If $x_{m}<0$ : the encrypted message is obtained using the following equation:

$\left\{\begin{array}{l}E M=F\left(m, x_{m}, z_{m}\right) \\ E M=p_{1} m+p_{2} x_{m}+p_{3} z_{m}\end{array}\right.$

where $\mathrm{m}$ is the clear message to be encrypted, $p_{1}, p_{2}$ and $p_{3}$ are constant parameters considered as a secret key in order to enhance complexity and confidentiality of the encryption process.

If $x_{m} \geq 0$ : the encrypted message is obtained using the following equation:

$\left\{\begin{array}{l}E M=Q\left(m, x_{m}, w_{m}\right) \\ E M=k_{1} m+k_{2} x_{m}+k_{3} w_{m}\end{array}\right.$

where $k_{1}, k_{2}$ and $k_{3}$ are constant parameters considered as a secret key in order to enhance complexity and confidentiality of the encryption process.

In the receiver, after achieving synchronization between the master system and the slave system, the decrypted message $D M$ is obtained by switching between two decrypted processes according to the value of the slave system first state $x_{s}$ using the following algorithm:

If $x_{s}<0$ : the decrypted message is obtained using the following equation: 


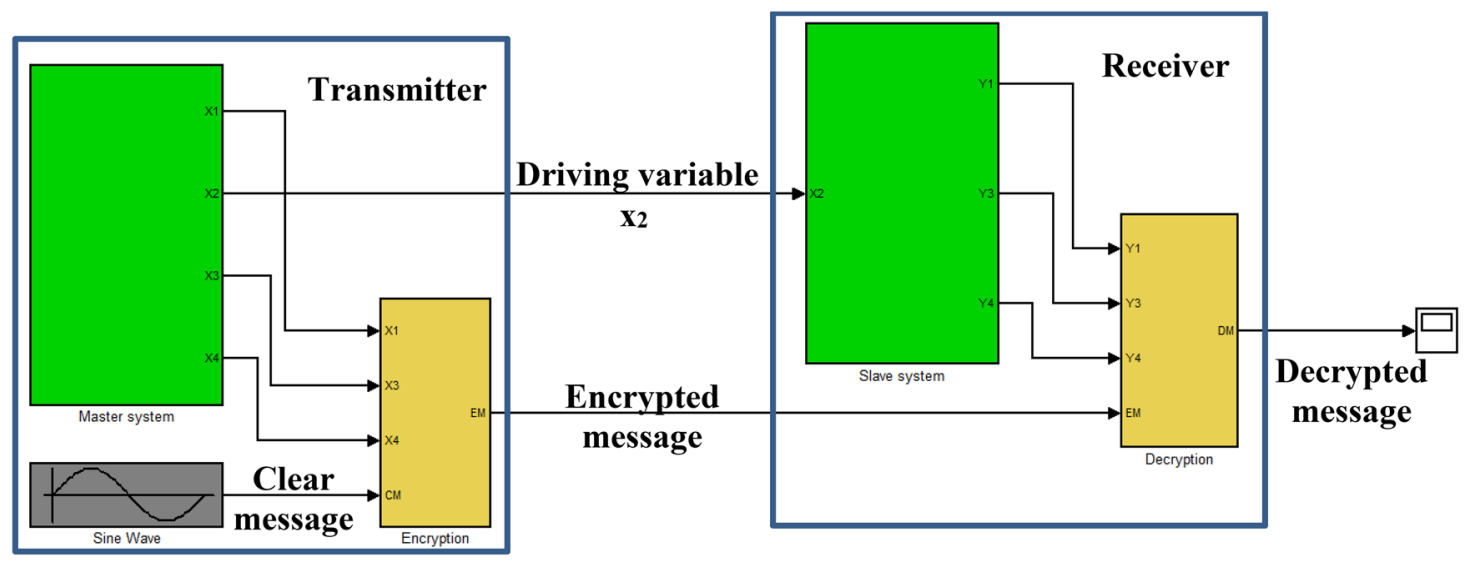

(a) The new secure communication scheme

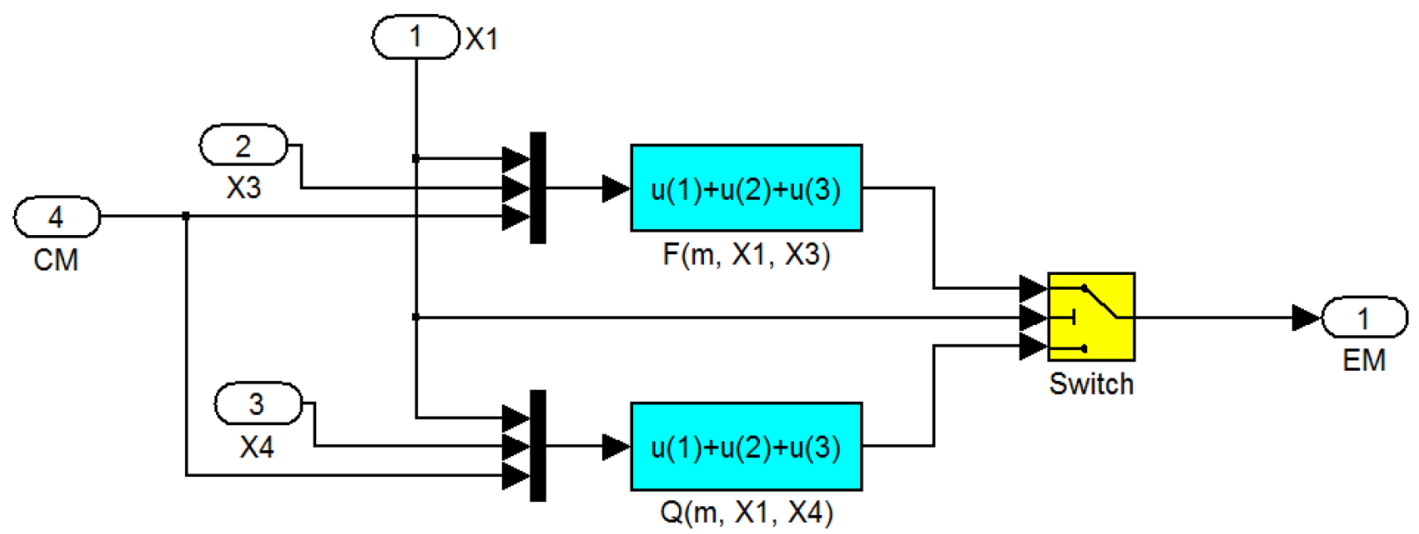

(b) Encryption process

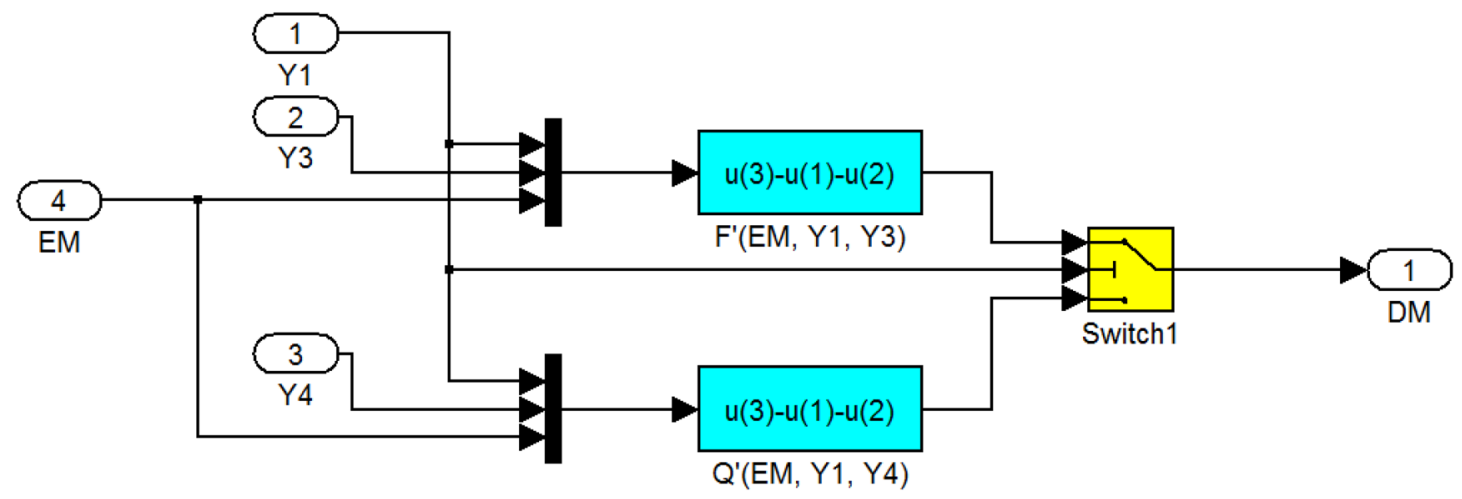

(c) Decryption process

Fig. 21 Secure communication Matlab/Simulink block diagram based on the new snail-shaped chaotic system 


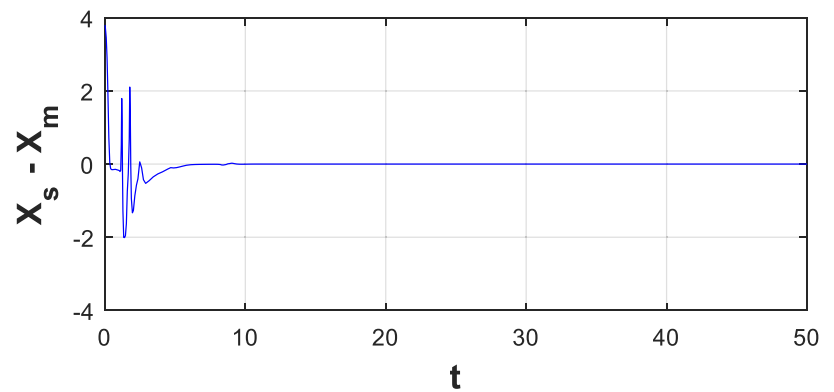

Fig. 22 Synchronization error of the first coordinates

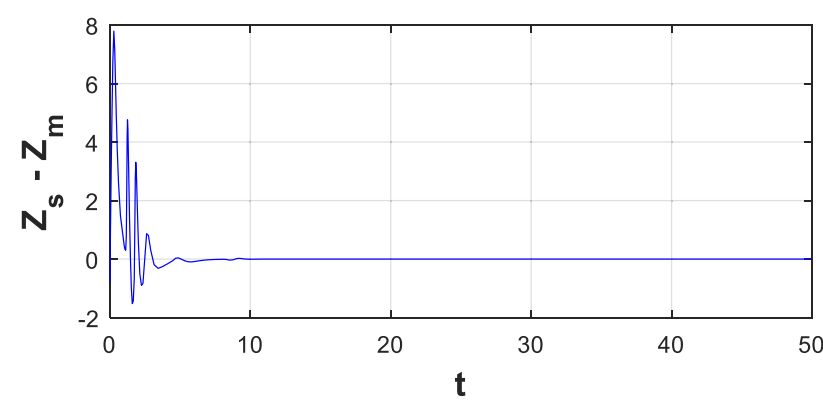

Fig. 23 Synchronization error of the third coordinates

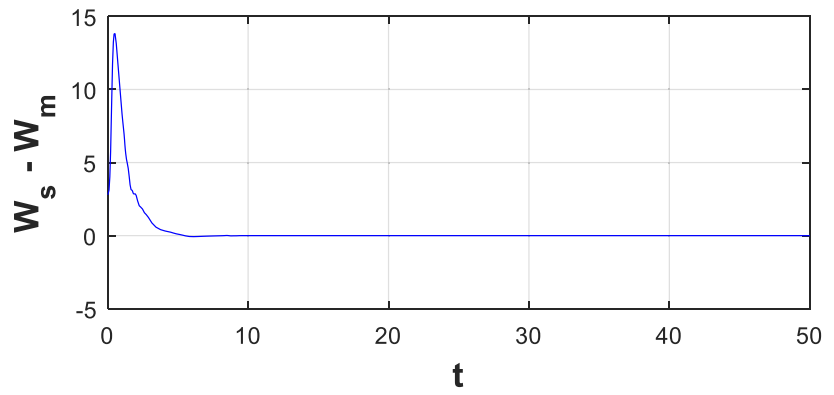

Fig. 24 Synchronization error of the fourth coordinates

$\left\{\begin{array}{l}D M=F^{\prime}\left(m, x_{s}, z_{s}\right) \\ D M=\frac{1}{p_{1}}\left(E M-p_{2} x_{s}-p_{3} z_{s}\right)\end{array}\right.$

where $E M$ is the encrypted message to be decrypted, $p_{1}, p_{2}$ and $p_{3}$ are the same constant parameters as in the transmitter.

If $x_{s} \geq 0$ : the decrypted message is obtained using the following equation:

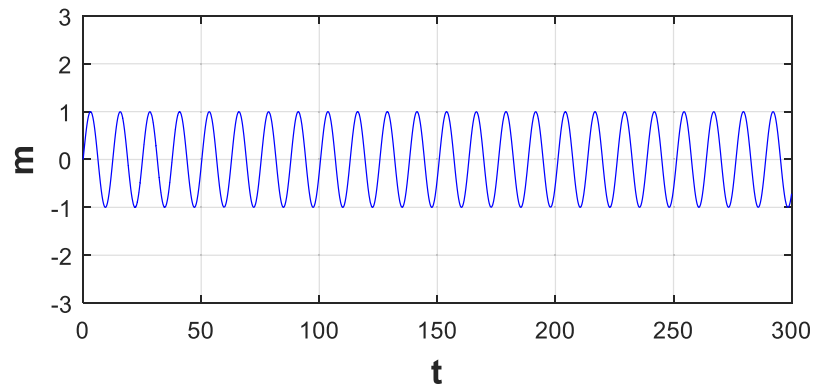

Fig. 25 Time evolution of the clear message

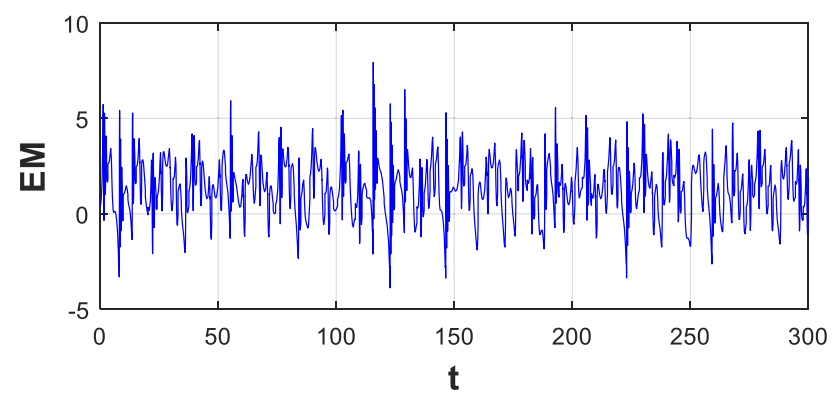

Fig. 26 Time evolution of the encrypted message

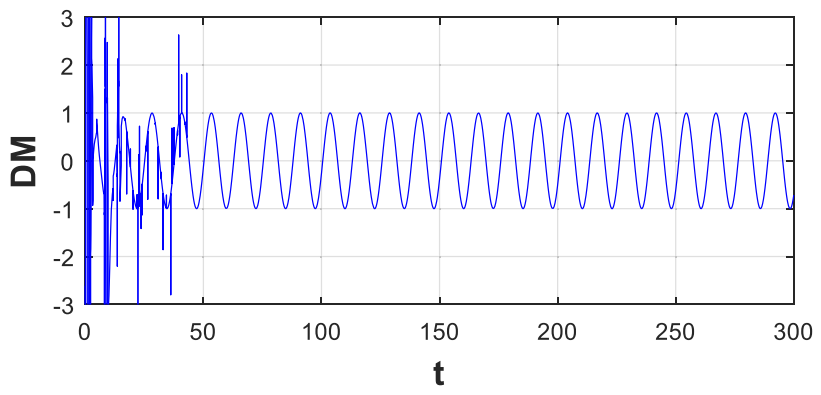

Fig. 27 Time evolution of the decrypted message

$\left\{\begin{array}{l}D M=Q^{\prime}\left(E M, x_{s}, w_{s}\right) \\ D M=\frac{1}{k_{1}}\left(E M-k_{2} x_{s}-k_{3} w_{s}\right)\end{array}\right.$

where $k_{1}, k_{2}$ and $k_{3}$ are the same constant parameters as in the transmitter.

- For numerical simulation

We chose the constant parameters of the encryption and the decryption functions as the following:

$P_{1}=P_{2}=P_{3}=k_{1}=k_{2}=k_{3}=1$ 


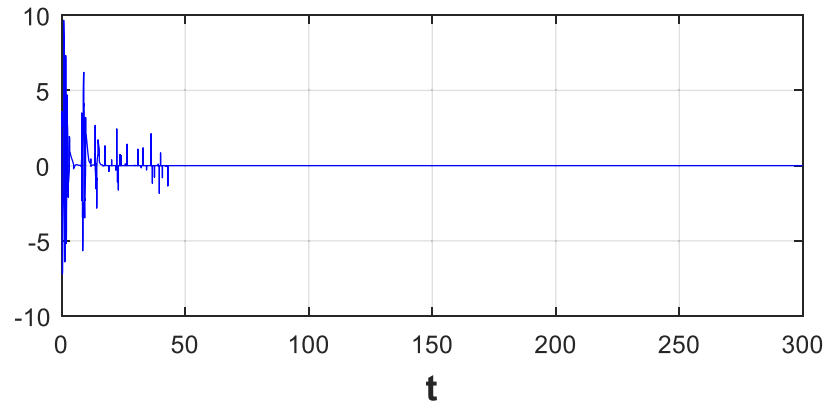

Fig. 28 Message reconstruction error

We choose the sine wave as the clear message to be transmitted as depicted in Fig. 25. Figure 26 shows the complex chaotic behavior of the encrypted message which confirm the effectiveness of the encrypting process to hide the clear message. The decrypted message is depicted in Fig. 27, we can see that after a few time (after achieving synchronization between master and slave systems) the obtained message is the same as the original message. Figure 28 shows the convergence of the message reconstruction error to zero after achieving synchronization, which confirms the success of the decryption process to reconstruct the original message.

\section{Conclusion}

In this work, a new snail-shaped chaotic system having eleven terms and four parameters is first presented. The new system can exhibit periodic, quasi-periodic and complex chaotic behaviors which are studied and proved theoretically by an exhaustive dynamical analysis and physically by implementing an equivalent electronic circuit via Multisim software. The new system is more complex and has a larger bandwidth than at least 30 recently reported chaotic systems, which is make it very useful in many fields of science such as: secure transmission and cryptography.

Also, synchronization between the novel system and the famous Rossler hyperchaotic system is achieved in order to illustrate the possibility of using the new system in applications, not only with chaotic systems but also with hyperchaotic systems. Finally, in order to test the applicability of the snail-shaped chaotic system a new secure communication scheme is developed for it by using drive response synchronization; simulation results proved the success of the proposed system to complete the secure transmission/reception process.

We strongly believe that the proposed chaotic system with its large bandwidth and complex behavior is desirable to use in a real secure transmission schemes in the near future.

\section{Compliance with ethical standards}

Conflict of interest The authors declare that they have no conflict of interests.

\section{References}

1. Vaseghi B, Pourmina MA, Mobayen S (2017) Secure communication in wireless sensor networks based on chaos synchronization using adaptive sliding mode control. Nonlinear Dyn 89(3):1689-1704

2. Kocamaz UE, Çiçek S, Uyaroğlu Y (2018) Secure communication with chaos and electronic circuit design using passivity-based synchronization. J Circuits Syst Comput 27(04):1850057

3. Benkouider K, Halimi M, Bouden T (2019) Secure communication scheme using chaotic time-varying delayed system. Int J Comput Appl Technol 60(2):175

4. Pham VT, Jafari S, Volos C (2017) A novel chaotic system with heart-shaped equilibrium and its circuital implementation. Optik (Stuttgart) 131:343-349

5. Abdolmohammadi HR, Khalaf AJM, Panahi S, Rajagopal K, Pham V-T, Jafari S (2018) A new 4D chaotic system with hidden attractor and its engineering applications: analog circuit design and field programmable gate array implementation. Pramana 90(6):70

6. Benkouider K, Bouden T, Halimi M (2019) Dynamical analysis, synchronization and circuit implementation of a new hyperchaotic system with line equilibrium. In: 20196 th international conference on control, decision and information technologies (CoDIT), pp 1717-1722

7. Lorenz EN (1963) Deterministic nonperiodic flow. J Atmos Sci 20:130

8. Zhang L (2017) A novel 4-D butterfly hyperchaotic system. Optik (Stuttgart) 131:215-220

9. Yu S, Lu J, Chen G (2007) Theoretical design and circuit implementation of multidirectional multi-torus chaotic attractors. IEEE Trans Circuits Syst I Regul Pap 54(9):2087-2098

10. Guo J, Xie G, Wang L (2009) Chaotic attractor generation and critical value analysis via switching approach. Chaos Solitons Fractals 40(5):2160-2169

11. Gan WS (1996) Techniques in the application of chaos theory in signal and image processing. In: Leondes $C$ (ed) Control and dynamic systems, vol 77, no C. Academic Press Inc., pp 339-387

12. Frederickson P, Kaplan JL, Yorke ED, Yorke JA (1983) The Liapunov dimension of strange attractors. J Differ Equ 49(2):185-207

13. Qi G, Chen G, Zhang Y (2008) On a new asymmetric chaotic system. Chaos Solitons Fractals 37(2):409-423

14. Chen G, Ueta T (1999) Yet another chaotic attractor. Int J Bifurc Chaos 09(07):1465-1466

15. Su K (2015) Dynamic analysis of a chaotic system. Optik (Stuttgart) 126(24):4880-4886

16. Akgul A, Hussain S, Pehlivan I (2016) A new three-dimensional chaotic system, its dynamical analysis and electronic circuit applications. Optik (Stuttgart) 127(18):7062-7071

17. Zhang $M$, Han $Q$ (2016) Dynamic analysis of an autonomous chaotic system with cubic nonlinearity. Optik (Stuttgart) 127(10):4315-4319

18. Gholamin P, Sheikhani AHR (2017) A new three-dimensional chaotic system: dynamical properties and simulation. Chin J Phys 55(4):1300-1309

19. Lai Q, Huang J, Xu G (2016) Coexistence of multiple attractors in a new chaotic system. Acta Phys Pol B 47:2315-2323 
20. Tuna M, Fidan CB (2016) Electronic circuit design, implementation and FPGA-based realization of a new 3D chaotic system with single equilibrium point. Optik (Stuttgart) 127(24):11786-11799

21. Wu X, He Y, Yu W, Yin B (2015) A new chaotic attractor and its synchronization implementation. Circuits Syst Signal Process 34(6):1747-1768

22. Volos C, Akgul A, Pham VT, Stouboulos I, Kyprianidis I (2017) A simple chaotic circuit with a hyperbolic sine function and its use in a sound encryption scheme. Nonlinear Dyn 89(2):1047-1061

23. Singh JP, Roy BK (2018) A more chaotic and easily hardware implementable new 3-D chaotic system in comparison with 50 reported systems. Nonlinear Dyn 93(3):1121-1148

24. Sambas A, Vaidyanathan S, Zhang S, Putra WT, Mamat M, Mohamed MA (2019) Multistability in a novel chaotic system with perpendicular lines of equilibrium: analysis, adaptive synchronization and circuit design. Eng Lett 27(4):1-8

25. Yang Q, Qiao X (2019) Constructing a new 3D chaotic system with any number of equilibria. Int J Bifurc Chaos 29(05):1950060

26. Lassoued A, Boubaker O, Dhifaoui R, Jafari S (2019) Experimental observations and circuit realization of a jerk chaotic system with piecewise nonlinear function. In: Boubaker O, Jafari S (eds) Recent advances in chaotic systems and synchronization. Elsevier, pp 3-21

27. Vaidyanathan S, AlhadjiAbba O, Betchewe G, Alidou M (2019) A new three-dimensional chaotic system: its adaptive control and circuit design. Int J Autom Control 13(1):101-121

28. Lien CH, Vaidyanathan S, Sambas A, Mamat M (2019) A new 3-D chaotic system with four quadratic nonlinear terms, its global chaos control via passive control method and circuit design. In: Tosida ET, Wihartiko D (eds) IOP conference series: materials science and engineering, vol 621, no 1. IOP Publishing

29. Kapitaniak T, Mohammadi S, Mekhilef S, Alsaadi F, Hayat T, Pham V-T (2018) A new chaotic system with stable equilibrium: entropy analysis, parameter estimation, and circuit design. Entropy 20(9):670

30. Xu G, Shekofteh Y, Akgül A, Li C, Panahi S (2018) A New Chaotic system with a self-excited attractor: entropy measurement, signal encryption, and parameter estimation. Entropy 20(2):86

31. Idowu BA, Vaidyanathan S, Sambas A, Olusola OI, Onma OS (2018) A new chaotic finance system: its analysis, control, synchronization and circuit design. In: Pham VT, Vaidyanathan S, Volos C, Kapitaniak T (eds) Studies in systems, decision and control, vol 133. Springer, Cham, pp 271-295
32. Jinjie $H$, Guangming $S$ (2018) A new chaotic system and its synchronization with phase spatial rotation. In: Proceedings of the 30th Chinese control and decision conference, CCDC 2018, pp 193-196

33. Lai Q, Akgul A, Varan M, Kengne J, Turan Erguzel A (2018) Dynamic analysis and synchronization control of an unusual chaotic system with exponential term and coexisting attractors. Chin J Phys 56(6):2837-2851

34. Sahin ME, Cam Taskiran ZG, Guler H, Hamamci SE (2020) Application and modeling of a novel 4D memristive chaotic system for communication systems. Circuits Syst Signal Process. https ://doi.org/10.1007/s00034-019-01332-6

35. Zhou C, Yang C, Xu D, Chen C (2020) Dynamic analysis and synchronisation control of a novel chaotic system with coexisting attractors. Pramana J Phys 94(1):19

36. Vaidyanathan $S$, Sambas $A$, Zhang $S$, Mujiarto AD, Mamat $M$, Subiyanto $S$ (2019) A chaotic jerk system with three cubic nonlinearities, dynamical analysis, adaptive chaos synchronization and circuit simulation. J Phys Conf Ser 1179:012083

37. Tasikmalaya UM, Mamat M, Arafa A, Mahmoud GM, Sambas A, Arafa AA, Mohamed MA, Sanjaya WSM (2019) A new chaotic system with line of equilibria: dynamics, passive control and circuit design. Artic Int J Electr Comput Eng 9(4):2365-2376

38. Wang L, Ding M (2018) Dynamical analysis and passive control of a new 4D chaotic system with multiple attractors. Mod Phys Lett B 32(22):1850260

39. Vaidyanathan S, Sambas A, Kacar S, Çavuşoglu Ü (2018) A new three-dimensional chaotic system with a cloud-shaped curve of equilibrium points, its circuit implementation and sound encryption. Int J Model Identif Control 30(3):184-196

40. Yanmin L, Fuhong $M$, Chuang L, Biaoming $Y$ (2018) A new fourdimensional four-wing chaotic circuit based on Qi chaotic system. In: Chinese control conference, CCC, vol 2018, pp 743-748

41. Zheng W, Zhang G (2019) Control and synchronization of a new class of finance chaotic systems. In: Proceedings of the 31st Chinese control and decision conference, CCDC 2019, pp 200-205

42. Benkouider K, Bouden T, Halimi M (2019) Analysis, circuit implementation and active control synchronization of a new 4D chaotic system with two quadratic nonlinearities. In: 2019 4th world conference on complex systems (WCCS), pp 1-6

Publisher's Note Springer Nature remains neutral with regard to jurisdictional claims in published maps and institutional affiliations. 